Original article

\title{
Histone modifications in Rett syndrome lymphocytes: a preliminary evaluation
}

\author{
Walter E. Kaufmann*, Mohammed H. Jarrar, Judy S. Wang, Ye-Jin M. Lee, \\ Sriram Reddy, Genila Bibat, Sakkubai Naidu \\ Kennedy Krieger Institute and Johns Hopkins University School of Medicine, 3901 Greenspring Avenue, Baltimore, MD 21205, USA
}

Received 13 April 2004; received in revised form 22 July 2004; accepted 10 September 2004

Abstract

Most cases of Rett syndrome (RTT) are associated with mutations in the coding region of the transcriptional regulator MeCP2. This gene appears to repress gene expression through chromatin conformational changes secondary to histone modifications, mainly histone deacetylation of core histones $\mathrm{H} 3$ and $\mathrm{H} 4$. There is limited and contradictory information about histone modifications in RTT tissues. The present study intended to provide a preliminary characterization of histone acetylation ( $\mathrm{AcH} 3$, AcH4) and methylation (MeH3) in RTT, with emphasis on non-selected peripheral cells and molecular-neurologic correlations. We compared 17 females with RTT, 11 of them with MeCP2 mutations, with 10 gender-matched controls in terms of lymphocyte lysate immunoblotting-based levels. We found that immunoreactivities for $\mathrm{MeCP} 2$ and $\mathrm{AcH} / \mathrm{AcH} 4$ are variable in both control and RTT subjects. Despite this variability, RTT subjects with nonsense mutations showed the expected reduction in C-terminal MeCP2 immunoreactivity. Regardless of MeCP2 levels, both subjects with (RTTPos) and without (RTTNeg) mutations had decreased levels of AcH3. The latter reductions were mainly driven by decreases in levels of $\mathrm{H} 3$ acetylated at lysine residue 14 (AcH3K14) and independent of parallel, but milder, decreases in immunoreactivity for MeH3 lysine residues (MeH3K4/MeH3K9). Within our study sample, reductions in $\mathrm{AcH} 3$ were correlated with severity of head growth deceleration in the RTTPos group. This contrasted with the lack of significant association between location of MeCP2 mutation and severity of the RTT neurologic phenotype. We concluded that there were distinctive profiles of histone acetylation/methylation in RTT peripheral cells, which reflect pathogenetic mechanisms common to subjects with clinical features of this disorder, regardless of mutation status, and that these patterns may be relevant to neurologic dysfunction in RTT.

(C) 2004 Published by Elsevier B.V.

Keywords: Rett syndrome; MeCP2; Histone; Acetylation; Neurologic impairment

\section{Introduction}

Rett syndrome (RTT) is a disorder that predominantly affects females and is characterized by severe cognitive impairment, autistic behavior, stereotypic movements, respiratory irregularities, and frequent seizures [1]. The majority of RTT cases are associated with mutations in the coding region of $M e C P 2$, a gene encoding a member of the family of methyl-binding proteins (MBDPs) [2-4]. As other MBDPs, MeCP2 contains a functional domain that

\footnotetext{
* Corresponding author. Tel.: +1 443923 2789; fax: +1 4439237615 .

E-mail address: kaufmann@kennedykrieger.org (W.E. Kaufmann).
}

binds to methylated $\mathrm{CpG}$ groups (MBD) and a second sequence (TRD) that is involved in transcriptional repression via a repressor complex that includes $\mathrm{mSin} 3 \mathrm{~A}$ and histone deacetylases [5]. Fuks and colleagues [6] have also reported a role for $\mathrm{MeCP} 2$ in promoting the methylation of histone H3. Post-translational modifications affecting lysine (K) and other residues in histone tails lead to changes in DNA-histone interactions and, consequently, to changes in chromatin configuration [7]. The balance between euchromatin ('active') and heterochromatin ('silent'), which ultimately determines whether a particular gene is transcribed or not, seems to depend on the post-translational balance of specific $\mathrm{K}$ residues, in particular, of histone $\mathrm{H} 3$ [7-9]. An example is provided by the silencing of $B D N F$ by 
$\mathrm{MeCP} 2$, which is associated with a relatively greater proportion of methylated $\mathrm{H} 3 \mathrm{~K} 9$ (MeH3K9) than acetylated H3K9 (AcH3K9) at the gene's promoter III [10].

There is considerable variability in the type and location of $\mathrm{MeCP} 2$ mutations in RTT patients. While missense mutations are predominantly located in the $\mathrm{MBD}$, nonsense mutations are typically in the TRD region [11]. This mutational heterogeneity is further complicated by the potential influence of skewed $\mathrm{X}$ chromosome inactivation, which can favor either the normal or the mutant allele [12]. Most MeCP2's functional in vitro assays, including DNA binding $[4,13,14]$, heterochromatin targeting $[15,16]$, and transcriptional repressive activity $[13,15,16]$, have evaluated mutations affecting the MBD. Histone acetylation has also been used as an index of MeCP2 dysfunction in a mutant mouse that models nonsense mutations in RTT; mice with the MeCP2 residue 308 truncation display RTT-like neurologic disturbances [17,18], increased $\mathrm{AcH} 3$ in brain and spleen, but normal neuronal heterochromatin targeting [17]. This and the aforementioned studies suggest that changes in histone modifications may be sensitive markers of MeCP2 dysfunction in RTT patients with all type of mutations. To date, only two studies have examined histone modifications in RTT samples. Wan et al. [19] found a selective increase in H4K16 acetylation in lymphoblasts from two individuals (one female RTT) with MeCP2 truncations. Another study found no changes in histone acetylation in T lymphocytes clones from RTT patients [20]. Discrepancies between these two studies may be due to cell cycle differences between transformed lymphoblasts and cloned lymphocytes. For this reason, evaluation of non-transformed, non-selected, peripheral lymphocytes in RTT may provide a more representative picture of the relationship between $\mathrm{MeCP} 2$ dysfunction and histone modifications, in particular in post-mitotic cells such as neurons. Lymphocytic histone profiles may also shed light on mutation negative RTT subjects (RTTNeg) and on the molecular events underlying $\mathrm{MeCP} 2$ mutations in patients with a non-RTT phenotype. Considering the limited genotype-phenotype correlations reported by several studies on RTT [12,21-32], histone modifications patterns have the potential to become informative molecular phenotypic indices. Consequently, we have performed a preliminary assessment of patterns of histone acetylation and methylation in lymphocyte samples from a group of females with classic RTT, and conducted an exploratory correlation of these histone parameters with neurologic severity.

\section{Material and methods}

\subsection{Subjects}

The present study included 17 female subjects with classic clinical features of RTT (RTTALL) and 10 control females (mean age 15.6 years, range 8.7-24.9 years). Eleven of these RTT patients (mean age 7.7 years, range 3.0-31.0 years) had mutations in $M e C P 2$ 's coding region (RTTPos), while 6 RTT girls (mean age 8.3 years, range 3.0-15.0 years) were RTTNeg. Among RTTPos subjects, the mutations spanned the MBD and TRD regions and consisted of one with a deletion (Del 796), eight with truncations (two R168X, one R255X, three R270X, one V288X, one R294X), and two with missense mutations (T158M, R306C). RTT subjects were recruited as part of a study on natural history and neurobiological correlates of RTT, while control subjects were participants in an investigation of the neurobiology of learning disabilities in girls with Fragile $\mathrm{X}$ syndrome. Informed consent was obtained from the subjects or legal guardians, meeting the standards of the Johns Hopkins Medical Institutions' institutional review board. Table 1 provides information about the RTT cohort and some basic molecular parameters.

\subsection{Genetic testing}

Genomic DNA was isolated from peripheral blood samples and $\mathrm{MeCP} 2$ mutations affecting exons 2, 3 and 4 and the $3^{\prime}$ UTR were evaluated by PCR, denaturing highpressure liquid chromatography (DHPLC), and direct automated sequencing as reported by Hoffbuhr et al. [27]. Following this, subjects were classified in terms of mutation as follows:

a. Location: mutations involving the TRD or C-terminal regions with respect to this domain were labeled as distal. All other mutations were considered proximal.

b. Type: mutations were divided into three categories: missense, nonsense or truncation, and C-terminal deletion as reported $[2,11,21]$.

c. Nuclear localization signal (NLS) involvement: mutations affecting the midportion of the TRD, which corresponds to the NLS, were considered as NLS Positive as published [28]. The remaining mutations were labeled as NLS Negative.

Description of mutation-related parameters in our RTT cohort is provided in Table 1 .

\subsection{Lymphocyte samples}

Histone analyses were also done on peripheral blood samples; leukocytes were separated using the CPT-Vacutainer system, which enriches the sample to approximately $80 \%$ lymphocytes [33]. Cells were lysed in a denaturing buffer (8 M urea, $0.2 \%$ SDS, $10 \% \beta$-mercaptoethanol, $10 \%$ glycerol, $62.5 \mathrm{mM} 0.5 \mathrm{M}$ Tris-Cl) as previously described [34,35]. 
Table 1

Characteristics of Rett syndrome subjects under study

\begin{tabular}{|c|c|c|c|c|c|c|c|}
\hline \multirow[t]{2}{*}{ Subject number } & \multirow[t]{2}{*}{ Age (yrs) } & \multirow[t]{2}{*}{ Mutation } & \multirow[t]{2}{*}{ Type of mutation ${ }^{\mathrm{a}}$} & \multirow{2}{*}{$\begin{array}{l}\text { Location of } \\
\text { mutation }^{\mathrm{a}}\end{array}$} & \multirow[t]{2}{*}{ NLS involvement ${ }^{\mathrm{a}}$} & \multicolumn{2}{|c|}{ MeCP2 level (OD) } \\
\hline & & & & & & $\overline{\mathrm{N} \text {-terminus }}$ & C-terminus \\
\hline RTT3066 & 2 & Del796 & Deletion & Distal & No & 122.00 & 163.28 \\
\hline RTT3062 & 3 & $\mathrm{R} 270 \mathrm{X}$ & Truncation & Distal & Yes & 149.55 & 90.72 \\
\hline RTT3051 & 3 & None & N/A & N/A & N/A & 80.50 & 140.34 \\
\hline RTT3064 & 3 & $\mathrm{R} 255 \mathrm{X}$ & Truncation & Distal & Yes & 193.45 & 73.02 \\
\hline RTT3068 & 4 & None & N/A & N/A & N/A & 122.10 & 254.81 \\
\hline RTT3049 & 4 & $\mathrm{R} 270 \mathrm{X}$ & Truncation & Distal & Yes & 349.50 & 116.18 \\
\hline RTT3056 & 4 & V288X & Truncation & Distal & Yes & 74.95 & 142.06 \\
\hline RTT3057 & 5 & $\mathrm{R} 306 \mathrm{C}$ & Missense & Distal & No & 95.25 & 179.82 \\
\hline RTT3065 & 6 & $\mathrm{R} 168 \mathrm{X}$ & Truncation & Proximal & N/A & 170.95 & 74.00 \\
\hline RTT2061 & 7 & $\mathrm{~T} 158 \mathrm{M}$ & Missense & Proximal & No & 157.05 & 140.55 \\
\hline RTT3048 & 8 & $\mathrm{R} 168 \mathrm{X}$ & Truncation & Proximal & Yes & 309.65 & 124.75 \\
\hline RTT3052 & 8 & None & N/A & N/A & N/A & 121.40 & 8.84 \\
\hline RTT3050 & 10 & $\mathrm{R} 294 \mathrm{X}$ & Truncation & Distal & No & 101.75 & 93.11 \\
\hline RTT3053 & 11 & None & N/A & N/A & N/A & 356.60 & 248.99 \\
\hline RTT3055 & 12 & None & N/A & N/A & N/A & 117.50 & 133.67 \\
\hline RTT1081 & 15 & None & N/A & N/A & N/A & 121.35 & 177.18 \\
\hline RTT2070 & 31 & $\mathrm{R} 270 \mathrm{X}$ & Truncation & Distal & Yes & 98.15 & 118.10 \\
\hline
\end{tabular}

$\mathrm{OD}$, arbitrary optical density units.

${ }^{\text {a }}$ N/A, not applicable.

\subsection{Molecular assays}

Samples were resolved by standard sodium dodecyl sulphate polyacrylamide gel electrophoresis (SDS-PAGE) using precast $4-20 \%$ gels (ISC BioExpress, Kaysville, UT, USA), transferred onto nitrocellulose membranes, immunoprobed, and visualized by the enhanced chemiluminescence (ECL) method, as described [34,35]. For detection and quantification of $\mathrm{MeCP} 2$ immunoreactivity, we used polyclonal antibodies (Abs) targeting either the N- or C-terminus of the protein [36]: an Ab directed to residues 9-27 of human MeCP2, kindly provided by Dr John Christodoulou (Children's Hospital at Westmead, Sydney, Australia), and an $\mathrm{Ab}$ that targets a highly conserved Cterminal epitope (i.e. residues 465-478) of mouse MeCP2 (Upstate Biotechnology, Waltham, MA, USA) [36-38]. Non-acetylated (also termed total) [17] and acetylated (Ac) $\mathrm{H} 3$ and $\mathrm{H} 4$, and di-methylated (Me) $\mathrm{H} 3$ were also detected by Abs supplied by Upstate. Antibodies recognized the following $\mathrm{H} 3$ and $\mathrm{H} 4$ modified residues: AcH3K9 and/or H3K14 ('pan' AcH3), any AcH4K residue (i.e. 'pan' AcH4: H4K5, H4K8, H4K12, and/or H4K16), AcH3K9, AcH3K14, AcH4K5, AcH4K8, AcH4K12, AcH4K16, $\mathrm{MeH} 3 \mathrm{~K} 4$, and MeH3K9. An actin Ab was obtained from Sigma (St Louis, MO, USA). Technical assays with lymphoblast whole cell lysates, and nuclear and cytoplasmic fractions (not shown) demonstrated that lysate-based measurements do correlate and are, therefore, representative of histone measurements in nuclear fractions.

Levels of MeCP2, H3, H4, and acetylated/methylated H3 and $\mathrm{H} 4$ were quantified as arbitrary optical density units by using the Molecular Dynamics Image Quant system (Amersham, Piscataway, NJ, USA) as published [36].

Levels of immunoreactivity were adjusted by quantifying protein load (intensity of Ponceau staining) and by calculating ratios to levels of actin.

\subsection{Neurologic and behavioral evaluations}

Control subject status was determined by standard neurologic examination, evaluation of global cognitive function (i.e. Wechsler Intelligence Scales [39], and exclusion of behavioral abnormalities and psychopathology (i.e. Achenbach Child Behavior Checklist [40], Diagnostic Interview for Children-Revised-Parent Version [41], Conners Rating Scale-Revised-Parent Version [42] as described in previous publications [43,44]. RTT patients were scored according to a Rett Syndrome Severity Scale (RSSS) through clinical examination or review of medical history as reported [27]. The RSSS evaluates five clinical features typical of RTT in a range of severity from 0 to 3: head growth (deceleration), frequency and manageability of seizures, respiratory irregularities, scoliosis, and ability to walk (gait apraxia), yielding total and feature-specific scores.

\subsection{Statistical analysis}

Our immunoblotting assays and statistical analyses followed the hierarchical approach reported by Wan et al. [19] for RTT lymphoblasts, which initially evaluated differences in pan (any $\mathrm{K}$ residue) acetylated $\mathrm{H} 3 / \mathrm{H} 4$ levels and, subsequently, changes in specific $\mathrm{K}$ residues of $\mathrm{H} 3 / \mathrm{H} 4$. As reported in the literature [17], in addition to absolute levels, we analyzed ratios of acetylated/nonacetylated $\mathrm{H} 3 / \mathrm{H} 4$. We not only recognized that $\mathrm{H} 3$ 


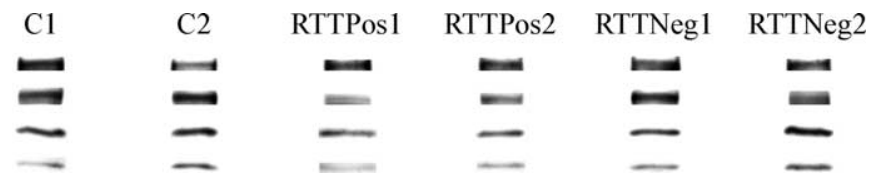

Fig. 1. Patterns of MeCP2 and acetylated histones $\mathrm{H} 3(\mathrm{AcH} 3)$ and $\mathrm{H} 4(\mathrm{AcH} 4)$ expression in RTT lymphocytes. Note the relatively comparable levels of immunoreactivity for N-terminal (Nt) and C-terminal MeCP2 epitopes in controls (C1, C2) and RTTNeg patients (RTTNeg1, RTTNeg2), which contrast with the lower C-terminal immunoreactivity in RTT patients with nonsense mutations (RTTPos1, RTTPos2). Moderate decreases in AcH3 levels were detected in RTT subjects, particularly in those with nonsense mutations (RTTPos1, RTTPos2). AcH4 levels were similar in control and RTT subjects.

acetylation status might be influenced by different acetylated $\mathrm{K}$ residues, but also by methylation of residues H3K4 and H3K9 [7-9]. Considering certain H3 modifications are linked to distinct chromatin configurations, specifically AcH3K9, AcH3K14, and MeH3K4 to euchromatin and MeH3K9 to heterochromatin [7-10,45], we also examined the relationship between these three modified $\mathrm{H} 3$ residues in each subject. Our molecular analyses first determined differences between RTTALL subjects and controls, followed by comparisons between the two RTT groups (RTTPos vs. RTTNeg). This approach was based on our conceptual framework, supported by extensive literature, that RTT is a clinical diagnosis which is independent of mutational status [46]. Consequently, RTT patients with and without $\mathrm{MeCP} 2$ mutations may share downstream molecular mechanisms. Taking into account that there were age differences between controls and RTT subjects (ANOVA $P=0.0003$ ), and a broader age range in the RTTNeg group, levels of specific proteins were compared by ANCOVAs (co-varying for age) using post-hoc analyses (i.e. Scheffe's) appropriate for small, unequal, and non-normally distributed samples, as previously reported [43]. Relationships between molecular variables were determined by simple and multiple linear regression analyses, including stepwise regression models for $\mathrm{AcH} 3$ specific residue-PanAcH3 analyses. Association between genotypic or molecular phenotypic (i.e. MeCP2, H3, H4) parameters and neurologic severity (RSSS) were also explored by linear regression models. Due to the RTT sample distribution, genotype-neurologic phenotype correlations were restricted to two categories: location of mutation and involvement of the NLS. All regression models introduced age as co-variate. Multiple comparisons were adjusted by the Bonferroni Multiple Comparison Procedure. All statistical analyses were conducted using Statview 5.0.1 ${ }^{\circledR}$.

\section{Results}

\subsection{MeCP2 levels}

In order to provide a baseline for the interpretation of the histone data, we first measured the levels of $\mathrm{MeCP} 2$ in all samples. We used two different Abs: a C-terminus Ab, for detection of reductions secondary to $\mathrm{C}$-terminal truncations or deletions (i.e. loss of epitope), and an $\mathrm{N}$-terminus $\mathrm{Ab}$ that detects both full length and truncated proteins [36]. In general, MeCP2 levels were variable in both control and RTT subjects (Fig. 1). Table 1 shows the values for each RTT patient, in addition to other basic information on the subjects. Table 2 presents the group data for controls and RTT patients; only assays with the C-terminus $\mathrm{Ab}$ demonstrated significantly lower MeCP2 levels in RTT subjects. This reduction was driven by the RTTPos group, which was mainly composed of patients with nonsense mutations (Table 1). No significant differences were found between RTTPos and RTTNeg patients for any MeCP2 $\mathrm{Ab}$ (Table 2).

\subsection{Levels of histones $H 3$ and $H 4$}

As in the case of $\mathrm{MeCP} 2$, levels of $\mathrm{H} 3$ and $\mathrm{H} 4$ immunoreactivity were variable. Although this variability was predominantly between subjects, we found a moderate $(\sim 20 \%)$ inter-assay variation. As expected, several technical factors appear to contribute to this inconsistency, the most critical being the signal-to-noise ratio of each $\mathrm{Ab}$. One RTTPos subject was excluded from these analyses because of virtually undetectable levels of NonAcH3 and NonAcH4. Considering that variability increases for parameters depending on more than one measure, such as ratios, we increased the alpha value for comparisons involving $\mathrm{H} 3$ or $\mathrm{H} 4$ ratios to $P=0.01$. Table 3 shows trend-level reductions in PanAcH3 ratios between both RTT groups and controls. Unexpectedly, while in the RTTPos group the decrease in $\mathrm{PanAcH} 3$ ratio was mainly the consequence of reductions in absolute levels of PanAcH3, in the RTTNeg cohort $\mathrm{PanAcH} 3$ ratio reductions were influenced by increased

Table 2

Levels of MeCP2

\begin{tabular}{lllll}
\hline & $\begin{array}{l}\text { N-terminus } \\
\left(\text { mean } \pm \mathrm{SE}^{\mathrm{a}}\right)\end{array}$ & $P$ & $\begin{array}{l}\text { C-terminus } \\
\left(\text { mean } \pm \mathrm{SE}^{\mathrm{a}}\right)\end{array}$ & $P$ \\
\hline Control & $204.8 \pm 49.5$ & 0.36 & $211.4 \pm 16.8$ & 0.002 \\
RTTALL & $161.2 \pm 21.9$ & & $134.0 \pm 14.8$ & \\
RTTPos & $165.6 \pm 26.8$ & 0.80 & $119.5 \pm 10.5^{\mathrm{b}}$ & 0.21 \\
RTTNeg & $153.2 \pm 41.2$ & & $160.6 \pm 37.0$ & \\
\hline
\end{tabular}

a $\mathrm{SE}$, standard error.

b Significant difference with respect to control group. 
Table 3

Levels of histone $\mathrm{H} 3$

\begin{tabular}{|c|c|c|c|c|c|c|}
\hline & $\begin{array}{l}\text { NonAcH3 } \\
\left(\text { mean } \pm \mathrm{SE}^{\mathrm{a}}\right)\end{array}$ & $P$ & $\begin{array}{l}\mathrm{PanAcH} 3 \\
\left(\text { mean } \pm \mathrm{SE}^{\mathrm{a}}\right)\end{array}$ & $P$ & $\begin{array}{l}\text { PanAcH3/NonAcH3 } \\
\left(\text { mean } \pm \mathrm{SE}^{\mathrm{a}}\right)\end{array}$ & $P$ \\
\hline Control & $851.7 \pm 368.5$ & 0.17 & $936.4 \pm 415.5$ & 0.08 & $3.69 \pm 2.14$ & 0.03 \\
\hline RTTALL & $1393.1 \pm 197.0$ & & $560.7 \pm 100.4$ & & $0.49 \pm 0.14$ & \\
\hline RTTPos & $1030.6 \pm 180.6$ & 0.009 & $379.1 \pm 62.1^{\mathrm{b}}$ & 0.01 & $0.52 \pm 0.21$ & 0.81 \\
\hline RTTNeg & $2045.7 \pm 265.3^{b}$ & & $863.5 \pm 199.4$ & & $0.44 \pm 0.11$ & \\
\hline
\end{tabular}

a SE, standard error.

b Significant difference with respect to control group.

Table 4

Levels of histone $\mathrm{H} 4$

\begin{tabular}{|c|c|c|c|c|c|c|}
\hline & $\begin{array}{l}\text { NonAcH4 } \\
\left(\text { mean } \pm \mathrm{SE}^{\mathrm{a}}\right)\end{array}$ & $P$ & $\begin{array}{l}\text { PanAcH4 } \\
\left(\text { mean } \pm \mathrm{SE}^{\mathrm{a}}\right)\end{array}$ & $P$ & $\begin{array}{l}\text { PanAcH4/NonAcH4 } \\
\left(\text { mean } \pm \mathrm{SE}^{\mathrm{a}}\right)\end{array}$ & $P$ \\
\hline Control & $820.6 \pm 232.6$ & 0.01 & $648.9 \pm 143.7$ & 0.18 & $0.96 \pm 0.33$ & 0.21 \\
\hline RTTALL & $341.6 \pm 62.3$ & & $483.3 \pm 56.5$ & & $3.01 \pm 1.06$ & \\
\hline RTTPos & $266.4 \pm 56.5^{b}$ & 0.10 & $422.4 \pm 63.3$ & 0.18 & $3.35 \pm 1.46$ & 0.72 \\
\hline RTTNeg & $476.8 \pm 129.3$ & & $584.8 \pm 101.1$ & & $2.39 \pm 1.55$ & \\
\hline
\end{tabular}

a SE, standard error.

b Significant difference with respect to control group.

levels of non-acetylated or total $\mathrm{H} 3$ (NonAcH3). Consequently, there were significant differences in levels of both PanAcH3 and NonAcH3 between RTTPos and RTTNeg patients. Table 4 illustrates the relative similarity in $\mathrm{H} 4$ parameters between RTT subjects and controls. Only levels of NonAcH4 were decreased in the RTT cohort, mainly at expense of the RTTPos group. These changes determined a mild, but not significant, increase in the PanAcH4 ratio in the RTTPos cohort.

\subsection{Acetylation of specific $\mathrm{H} 3$ lysine residues in RTT lymphocytes}

Since the most consistent changes in histone acetylation affected $\mathrm{H} 3$, and in following a scheme that led to the identification of a selective increase in AcH4K16 levels in RTT lymphoblasts [19], we examined the differential contribution of $\mathrm{AcH} 3 \mathrm{~K} 9$ and $\mathrm{AcH} 3 \mathrm{~K} 14$ to the reduction in $\mathrm{PanAcH} 3$ levels and ratio. We did not measure levels of AcH3K18 or AcH3K23, because to date there is no $\mathrm{Ab}$ that can detect acetylation of these residues in the context of AcH3K9 and/or AcH3K14. Moreover, most publications in the field refer to the AcH3K9/ $\mathrm{AcH} 3 \mathrm{~K} 14 \mathrm{Ab}$ applied here as the 'PanAcH3' Ab [17,19, 20]. As depicted in Table 5, in terms of ratios, we found a reduction in both $\mathrm{AcH} 3 \mathrm{~K} 9$ and $\mathrm{AcH} 3 \mathrm{~K} 14$ levels when any RTT group was compared with controls. The decreases were more pronounced, and of a comparable magnitude to those of PanAcH3, for AcH3K14. In agreement with these findings, linear regression analyses demonstrated that AcH3K14 was a better predictor of $\mathrm{PanAcH} 3$ levels than AcH3K9 contributing 89 and $69 \%$ of the variance in PanAcH3 for controls and RTT subjects, respectively, in models that included age as co-variate.

\subsection{Histone $H 3$ acetylation-methylation balance in RTT lymphocytes}

Recent studies have demonstrated the critical role that H3K9 plays in the balance between active and silent chromatin associated to specific genes [7-10,45,47], and the potential interaction between di-methylated residues $\mathrm{H} 3 \mathrm{~K} 4$ and $\mathrm{H} 3 \mathrm{~K} 9$ and $\mathrm{AcH} 3 \mathrm{~K} 9$ in determining $\mathrm{H} 3$ acetylation status and conformation [7-9]. Consequently, we compared the levels of MeH3K4 and MeH3K9 between RTT and control subjects and found a decrease in the two methylated modifications in both RTT groups, more marked for MeH3K4 (Table 6). We then examined regression models testing the relationship between all four $\mathrm{H} 3$ modifications (i.e. AcH3K9, AcH4K14, MeH3K4, MeH3K9) and PanAcH3 levels. Even after removing AcH3K14 from the models, there was no relationship between either methylated residue and PanAcH3. Nonetheless, MeH3K4 and MeH3K9 levels were highly correlated between themselves (MeH3K4 vs. MeH3K9, adj. $R$ squared 0.61, $P=0.0007$; MeH3K4 ratio vs. MeH3K9 ratio, adj. $R$ squared 0.30 , $P=0.02$; both models with age as co-variate).

Table 5

Levels of acetylated $\mathrm{H} 3$ residues

\begin{tabular}{|c|c|c|c|c|}
\hline & \multicolumn{2}{|c|}{$\mathrm{AcH} 3 \mathrm{~K} 9 / \mathrm{NonAcH} 3$} & \multicolumn{2}{|c|}{ AcH3K14/NonAcH3 } \\
\hline & Mean $\pm \mathrm{SE}^{\mathrm{a}}$ & $P$ & Mean $\pm \mathrm{SE}^{\mathrm{a}}$ & $P$ \\
\hline Control & $5.02 \pm 3.65$ & 0.07 & $2.90 \pm 1.18$ & 0.03 \\
\hline RTTALL & $0.46 \pm 0.12$ & & $0.86 \pm 0.25$ & \\
\hline RTTPos & $0.54 \pm 0.14$ & 0.39 & $0.82 \pm 0.35$ & 0.81 \\
\hline RTTNeg & $0.32 \pm 0.50$ & & $0.96 \pm 0.29$ & \\
\hline
\end{tabular}

a SE, standard error. 
Table 6

Levels of methylated $\mathrm{H} 3$ residues

\begin{tabular}{|c|c|c|c|c|}
\hline & \multicolumn{2}{|c|}{ MeH3K4/NonAcH3 } & \multicolumn{2}{|c|}{$\mathrm{MeH} 3 \mathrm{~K} 9 / \mathrm{NonAcH} 3$} \\
\hline & Mean $\pm \mathrm{SE}^{\mathrm{a}}$ & $P$ & Mean $\pm \mathrm{SE}^{\mathrm{a}}$ & $P$ \\
\hline Control & $0.145 \pm 0.089$ & 0.04 & $0.538 \pm 0.432$ & 0.09 \\
\hline RTTALL & $0.019 \pm 0.003$ & & $0.051 \pm 0.011$ & \\
\hline RTTPos & $0.023 \pm 0.004$ & 0.20 & $0.054 \pm 0.013$ & 0.74 \\
\hline RTTNeg & $0.014 \pm 0.004$ & & $0.046 \pm 0.020$ & \\
\hline
\end{tabular}

${ }^{a} \mathrm{SE}$, standard error.

\subsection{Relationship between age, MeCP2, and histones $\mathrm{H3}$ and $\mathrm{H} 4$ in RTT lymphocytes}

There was no relationship between age and MeCP2 levels. Among the major $\mathrm{H} 3 / \mathrm{H} 4$ variables, age-dependent trends were determined by outliers in either control or RTT groups. There was no significant correlation between $\mathrm{N}$-terminus or C-terminus MeCP2 immunoreactivity and either PanAcH3 or PanAcH4 for any group, although the RTTPos cohort showed a weak inverse association between MeCP2 C-terminal immunoreactivity and PanAcH3 levels.

\subsection{Mutation parameters, histone levels, and neurologic severity in $R T T$}

The first set of preliminary correlational analyses contrasted mutation parameter-neurologic phenotype with molecular phenotype-neurologic phenotype associations within the RTTPos group. Only two mutation-related variables, namely location of mutation and involvement of the NLS, were analyzed. Considering the apparent lack of relationship between levels of methylated $\mathrm{H} 3$ residues and $\mathrm{AcH} 3$ immunoreactivity, we evaluated PanAcH3 and MeH3K4/MeH3K9 levels in terms of correlations separately. As shown in Table 7, there was no correlation between any of the two mutation parameters and RSSS scores. Similarly, the decreased C-terminal MeCP2 immunoreactivity was not associated with any RSSS variable in the RTTPos cohort. In contrast, both absolute PanAcH3

Table 7

Mutation parameters, MeCP2, acetylated $\mathrm{H} 3$, and neurologic severity in RTT

\begin{tabular}{|c|c|c|c|c|c|c|}
\hline & Composite & Head growth ${ }^{\mathrm{a}}$ & Seizures $^{\mathrm{a}}$ & $\begin{array}{l}\text { Respiratory } \\
\text { irregularities }^{\text {a }}\end{array}$ & Scoliosis $^{\mathrm{a}}$ & Ability to walk ${ }^{\mathrm{a}}$ \\
\hline Location of Mutation & NS & NS & NS & NS & NS & NS \\
\hline NLS Involvement & NS & NS & NS & NS & NS & NS \\
\hline MeCP2 (C-terminus IR) & NS & NS & NS & NS & NS & NS \\
\hline PanAcH3 & NS & $\begin{array}{l}\text { Trend (I) } \\
P=0.02\end{array}$ & NS & NS & $\begin{array}{l}\text { Trend (D) } \\
P=0.03\end{array}$ & $\begin{array}{l}\text { Significant (I) } \\
P=0.01\end{array}$ \\
\hline Pan $\mathrm{AcH} 3$ ratio & $\begin{array}{l}\text { Trend (I) } \\
P=0.10\end{array}$ & $\begin{array}{l}\text { Significant (I) } \\
P=0.005\end{array}$ & NS & NS & $\begin{array}{l}\text { Significant (D) } \\
P<0.0001\end{array}$ & NS \\
\hline $\mathrm{MeH} 3 \mathrm{~K} 4$ & $\begin{array}{l}\text { Trend (D) } \\
(P=0.07)\end{array}$ & NS & NS & NS & NS & NS \\
\hline МeH3К9 & $\begin{array}{l}\text { Trend (D) } \\
(P=0.07)\end{array}$ & NS & NS & NS & NS & NS \\
\hline
\end{tabular}

All analyses: age as a co-variate. (I): inverse relationship. (D): direct relationship.

${ }^{a}$ Alpha value after Bonferroni correction, $P \leq 0.01$. levels and PanAcH3 ratios were inversely correlated with deceleration in head growth and directly related to severity of scoliosis. There was also a weak direct association between immunoreactivity for MeH3K4 and MeH3K9 and RSSS composite scores. Since our main molecular finding was a reduction in PanAcH3 levels in RTT lymphocytes, the biological significance of an inverse correlation between AcH3 and head growth seems evident. The direct relationship between $\mathrm{H} 3$ parameters, either $\mathrm{AcH} 3$ or MeH3K4/MeH3K9, and RSSS scores is at this point of unclear biological significance. The validity of the AcH3 findings was corroborated by regression models, in which the three main molecular phenotype parameters (i.e. $\mathrm{MeCP} 2, \mathrm{AcH} 3, \mathrm{AcH} 4)$ were compared in terms of their influence upon RSSS scores. Again, only AcH3 predicted deceleration in head growth, contributing $40-65 \%$ of the variance in scores. Evaluations of the two consistent $\mathrm{AcH} 3-$ RSSS score associations (i.e. head growth, scoliosis) in the entire RTT cohort demonstrated that these correlations were driven by the RTTPos group.

\section{Discussion}

MeCP2 appears to repress transcription through chromatin conformational changes secondary to histone modifications [3-5,10,45]. MeCP2s dysfunction in RTT would lead to an increase in histone acetylation and gene expression. However, data on histone modifications and transcription in RTT tissues have been limited and, to some extent, contradictory $[17,19,20,48,49]$. The present study intended to examine, in non-selected peripheral cells, patterns of histone modifications and their possible association with neurologic involvement in 17 females with RTT. We found that, in lymphocytes from both control and RTT subjects, levels of MeCP2 and acetylated histones are variable. Despite this variability, RTTPos subjects with nonsense mutations showed the expected selective reductions in $\mathrm{C}$-terminal $\mathrm{MeCP} 2$ immunoreactivity. Both RTTPos and RTTNeg patients had decreased levels of 
$\mathrm{AcH} 3$, mainly driven by reductions in $\mathrm{AcH} 3 \mathrm{~K} 14$ levels that were parallel to milder decreases in immunoreactivity for methylated $\mathrm{H} 3 \mathrm{~K}$ residues. Within our study sample, reductions in $\mathrm{AcH} 3$ were correlated with severity of head growth deceleration in the RTTPos group that contrasted with the lack of significant association between location of MeCP2 mutation and severity of the RTT neurologic phenotype.

To our knowledge, only two studies have examined post-translational changes affecting histones in RTT cells. While an initial study found a selective increase in AcH4K16 in lymphoblasts from a female RTT patient with a common MeCP2 truncation (i.e. 168X) and from a male hemizygous for a TRD truncation [19], a second investigation on cloned $\mathrm{T}$ lymphocytes from 4 RTT subjects with different $\mathrm{MeCP} 2$ mutations reported no changes in levels of $\mathrm{AcH} 3$ or $\mathrm{AcH} 4$ [20]. A third study, characterizing a mouse model with a MeCP2 truncation, demonstrated an increase in $\mathrm{AcH} 3$ in several brain regions and spleen but not in liver [17]. Our data show that, either as absolute level (only RTTPos) or ratio to total H3 (entire RTT cohort), there is a decrease in AcH3 in RTT lymphocytes. This rather unexpected finding was supported and extended by the analyses of levels, and ratios, of acetylated $\mathrm{H} 3$ residues. The consistency of the pan (any $\mathrm{K}$ residue) and specific $\mathrm{K}$ residue measurements makes technical factors unlikely contributors to these findings. Cell/tissue- and cell cycle-related factors are the most suitable explanation for the unanticipated reduction in $\mathrm{AcH} 3$, as suggested by the tissue differences in the abovementioned mouse study [17] and by our inability to detect, as previously reported in lymphoblasts [19], AcH4K5 immunoreactivity in control or RTT lymphocyte lysates. Although MeCP2 levels were variable, the patterns of $\mathrm{N}$ - and C-terminal immunoreactivity were those predicted by the type of $M e C P 2$ mutation; only the RTTPos group (mainly nonsense mutations) displayed a decrease in C-terminal MeCP2 levels. Correspondingly, there was a weak relationship between $\mathrm{MeCP} 2$ deficit and reduced $\mathrm{AcH} 3$ levels in the RTTPos cohort.

Recent publications have emphasized the role of the balance in $\mathrm{H} 3$ residue modifications in determining chromatin configuration and gene expression [8,9]. In this first analysis of H3 methylation in RTT tissues, we found minimal changes in MeH3K9 levels despite studies showing an increase in the MeH3K9/AcH3K9 ratio in the MeCP2-mediated silencing of hypermethylated FMR1 $[45,47]$ and $B D N F$ [10] and the potential role of MeCP2 in promoting $\mathrm{H} 3 \mathrm{~K} 9$ methylation [6]. Unexpectedly, levels of the active chromatin-linked MeH3K4 were significantly decreased. Further emphasizing the complexity of our histone modifications findings is the fact that reductions in $\mathrm{AcH} 3$ immunoreactivity were driven by decreases in AcH3K14. The latter modification, which is coupled to phosphorylation of H3's serine 10 [50,51], appears to be a direct link between signaling cascades and gene expression particularly in neurons [52,53]. In line with this, different cell stimuli can induce complex profiles of histone changes that include among others parallel reductions in MeH3K9 and MeH3K4 [47,51]. The relative independence of $\mathrm{H} 3$ and $\mathrm{H} 4$ acetylation and $\mathrm{H} 3$ methylation is also suggested by the differential effects of inhibitors of DNA methylation or histone deacetylation on these post-translational changes [45,47,51]. Although the functional significance of histone modifications at the whole nucleus level is still uncertain [54], the histone profiles reported here suggest that in RTT lymphocytes H3 changes may be contributed by factors other than $\mathrm{MeCP} 2$ dysfunction. The conceptual framework of the present study, that the phenotypical features that define RTT are the result of a set of molecular events commonly but not necessarily linked to $M e C P 2$ mutations, was supported by our demonstration of similar histone profiles in RTTPos and RTTNeg patients. A recent study showing changes in $\mathrm{MeCP} 2$ immunoreactivity in post-mortem brain samples from subjects with idiopathic autism, and Angelman and Prader-Willi syndromes [55], indicates that patterns of histone modifications may be also be informative in other developmental disorders.

To date, at least 11 major genotype-phenotype studies in RTT have led to conflicting results [12,21-32]. Multiple factors, including variability in sample size and phenotypical measures and profiles of $\mathrm{X}$ chromosome inactivation distribution, may explain the discrepancies. Our preliminary histone profile-neurologic severity analyses indicate that, levels of acetylated $\mathrm{H} 3$ appear to be better indicators of neurologic severity than location of mutation or MeCP2 levels in RTTPos patients. Reductions in AcH3 levels were inversely correlated in particular with deceleration of head growth, a major measure of RTT neurologic involvement [56] that in large samples has been correlated to MBD location and missense type of mutation [30]. Since the majority of our RTTPos patients had nonsense mutations, we could not compare the latter genotypic parameters with histone patterns.

The present study should be considered as an initial examination of the potential usefulness of patterns of histone modifications, in non-selected and highly accessible peripheral cells, in RTT. Despite limitations such as sample size, underrepresentation of missense mutations, restricted control-RTT age-matching, and small number of phenotypical parameters, our data suggest that lymphocyte histone profiles may become valuable markers of dynamic nuclear events in the spectrum of patients with RTT and/or $M e C P 2$ mutations. Patterns of histone post-translational changes may also complement standard genotype-phenotype correlations. Follow-up studies with larger samples, comparative analyses with neural tissues, and correlations with a wider range of neurobehavioral features are necessary to fully evaluate the significance of histone post-translational modifications in RTT lymphocytes. 


\section{Acknowledgements}

We thank J. Christodoulou for supplying MeCP2 antibodies, M. Johnston and S. Baylin for advice and encouragement throughout the project, and T. Jansen for editorial assistance. We are also grateful to the families of our research participants, and in particular to our subjects with Rett syndrome. This work was supported by NIH grants HD24448 and HD24061, and the FRAXA Research Foundation.

\section{References}

[1] Naidu S. Rett syndrome: a disorder affecting early brain growth. Ann Neurol 1997;42:3-10.

[2] Van den Veyver IB, Zoghbi HY. Mutations in the gene encoding methyl-CpG-binding protein 2 cause Rett syndrome. Brain Dev 2001; 23:S147-S51.

[3] Nan X, Campoy FJ, Bird A. MeCP2 is a transcriptional repressor with abundant binding sites in genomic chromatin. Cell 1997;88:471-81.

[4] Ballestar E, Wolfe AP. Methyl-CpG binding proteins: targeting specific gene repression. Eur J Biochem 2001;268:1-6.

[5] Wade PA. Methyl CpG-binding proteins and transcriptional repression. Bioessays 2001;23:1131-7.

[6] Fuks F, Hurd PJ, Wolf D, Nan X, Bird AP, Kouzarides T. The methylCpG-binding protein MeCP2 links DNA methylation to histone methylation. J Biol Chem 2003;278:4035-40.

[7] Jenuwein T, Allis CD. Translating the histone code. Science 2001; 293:1074-80.

[8] Dillon N, Festenstein R. Unravelling heterochromatin: competition between positive and negative factors regulates accessibility. Trends Genet 2002;18:252-8. Eliminate.

[9] Turner BM. Cellular memory and the histone code. Cell 2002;111: $285-91$.

[10] Chen WG, Chang Q, Lin Y, Meissner A, West AE, Griffith EC, et al. Derepression of BDNF transcription involves calcium-dependent phosphorylation of MeCP2. Science 2003;302:885-9.

[11] Lee SS, Wan M, Francke U. Spectrum of MECP2 mutations in Rett syndrome. Brain Dev 2001;23:S138-S43.

[12] Hoffbuhr KC, Moses LM, Jerdonek MA, Naidu S, Hoffman EP. Associations between MeCP2 mutations, X-chromosome inactivation, and phenotype. Ment Retard Dev Disabil Res Rev 2002;8: 99-105.

[13] Yusufzai TM, Wolffe AP. Functional consequences of Rett syndrome mutations on human MeCP2. Nucleic Acids Res 2000;28:4172-9.

[14] Free A, Wakefield RI, Smith BO, Dryden DT, Barlow PN, Bird AP. DNA recognition by the methyl-CpG binding domain of $\mathrm{MeCP} 2$. J Biol Chem 2001;276:3353-60.

[15] Kudo S, Nomura Y, Segawa M, Fujita N, Nakao M, Dragich J, et al. Functional analyses of MeCP2 mutations associated with Rett syndrome using transient expression systems. Brain Dev 2001;23: S165-S73.

[16] Kudo S, Nomura Y, Segawa M, Fujita N, Nakao M, Schanen C, et al. Heterogeneity in residual function of $\mathrm{MeCP} 2$ carrying missense mutations in the methyl CpG binding domain. J Med Genet 2003;40: 487-93.

[17] Shahbazian M, Young J, Yuva-Paylor L, Spencer C, Antalffy B, Noebels J, et al. Mice with truncated MeCP2 recapitulate many Rett syndrome features and display hyperacetylation of histone $\mathrm{H} 3$. Neuron 2002;35:243-54.

[18] Young JI, Zoghbi HY. X-chromosome inactivation patterns are unbalanced and affect the phenotypic outcome in a mouse model of Rett syndrome. Am J Hum Genet 2004;74:511-20.
[19] Wan M, Zhao K, Lee SS, Francke U. MECP2 truncating mutations cause histone $\mathrm{H} 4$ hyperacetylation in Rett syndrome. Hum Mol Genet 2001;10:1085-92.

[20] Balmer D, Arredondo J, Samaco RC, LaSalle JM. MECP2 mutations in Rett syndrome adversely affect lymphocyte growth, but do not affect imprinted gene expression in blood or brain. J Hum Genet 2002; 110:545-52.

[21] Amano K, Nomura Y, Segawa M, Yamakawa K. Mutational analysis of the MECP2 gene in Japanese patients with Rett syndrome. J Hum Genet 2000;45:231-6.

[22] Amir RE, Van den Veyver IB, Schultz R, Malicki DM, Tran CQ, Dahle EJ, et al. Influence of mutation type and $\mathrm{X}$ chromosome inactivation on Rett syndrome phenotypes. Ann Neurol 2000;47: 670-9.

[23] Bienvenu T, Carrie A, de Roux N, Vinet MC, Jonveaux P, Couvert P, et al. MECP2 mutations account for most cases of typical forms of Rett syndrome. Hum Mol Genet 2000;9:1377-84.

[24] Cheadle JP, Gill H, Fleming N, Maynard J, Kerr A, Leonard H, et al. Long-read sequence analysis of the MECP2 gene in Rett syndrome patients: correlation of disease severity with mutation type and location. Hum Mol Genet 2000;9:1119-29.

[25] Huppke P, Laccone F, Kramer N, Engel W, Hanefeld F. Rett syndrome: analysis of MECP2 and clinical characterization of 31 patients. Hum Mol Genet 2000;9:1369-75.

[26] Auranen M, Vanhala R, Vosman M, Levander M, Varilo T, Hietala M, et al. MECP2 gene analysis in classical Rett syndrome and in patients with Rett-like features. Neurology 2001;56:611-7.

[27] Hoffbuhr K, Devaney JM, LaFleur B, Sirianni N, Scacheri C, Giron J, et al. MeCP2 mutations in children with and without the phenotype of Rett syndrome. Neurology 2001;56:1486-95.

[28] Huppke P, Held M, Hanefeld F, Engel W, Laccone F. Influence of mutation type and location on phenotype in 123 patients with Rett syndrome. Neuropediatrics 2002;33:63-8.

[29] Chae JH, Hwang YS, Kim KJ. Mutation analysis of MECP2 and clinical characterization in Korean patients with Rett syndrome. J Child Neurol 2002;17:33-6.

[30] Weaving LS, Williamson SL, Bennetts B, Davis M, Ellaway CJ, Leonard $\mathrm{H}$, et al. Effects of MECP2 mutation type, location and $\mathrm{X}$ inactivation in modulating Rett syndrome phenotype. Am J Med Genet 2003;118A:103-14.

[31] Naidu S, Bibat G, Kratz L, Kelley RI, Pevsner J, Hoffman E, et al. Clinical variability in Rett syndrome. J Child Neurol 2003;18:662-8.

[32] Leonard H, Colvin L, Christodoulou J, Schiavello T, Williamson S, Davis M, et al. Patients with the R133C mutation: is their phenotype different from patients with Rett syndrome with other mutations? J Med Genet 2003;40:e52.

[33] Mole L, Margolis D, Carroll R, Todd J, Holodniy M. Stabilities of quantitative plasma culture for human immunodeficiency virus, RNA, and p24 antigen from samples collected in Vacutainer CPT and standard Vacutainer tubes. J Clin Microbiol 1994;32:2212-5.

[34] Kaufmann WE, Abrams MT, Chen W, Reiss AL. Genotype, molecular phenotype, and cognitive phenotype: correlations in fragile X syndrome. Am J Med Genet 1999;83:286-95.

[35] Sun HT, Cohen S, Kaufmann WE. Annexin-1 is abnormally expressed in fragile $\mathrm{X}$ syndrome: two dimensional electrophoresis study in lymphocytes. Am J Med Genet 2001;103:81-90.

[36] Jarrar MH, Danko CG, Reddy S, Lee YM, Bibat G, Kaufmann WE. $\mathrm{MeCP} 2$ expression in human cerebral cortex and lymphoid cells: immunochemical characterization of a novel higher molecular weight form. J Child Neurol 2003;18:675-82.

[37] LaSalle JM, Goldstine J, Balmer D, Greco CM. Quantitative localization of heterogeneous methyl-CpG-binding protein 2 (MeCP2) expression phenotypes in normal and Rett syndrome brain by laser scanning cytometry. Hum Mol Genet 2001;10:1729-40.

[38] Aber KM, Nori P, MacDonald S, Bibat G, Jarrar MH, Kaufmann WE. Methyl-CpG-binding protein 2 is localized in the postsynaptic 
[48] Tudor M, Akbarian S, Chen RZ, Jaenisch R. Transcriptional profiling of a mouse model for Rett syndrome reveals subtle transcriptional changes in the brain. Proc Natl Acad Sci USA 2002; 99:15536-41.

[49] Traynor J, Agarwal P, Lazzeroni L, Francke U. Gene expression patterns vary in clonal cell cultures from Rett syndrome females with eight different MECP2 mutations. BMC Med Genet 2002;3:12.

[50] Cheung P, Tanner KG, Cheung WL, Sassone-Corsi P, Denu JM, Allis CD. Synergistic coupling of histone $\mathrm{H} 3$ phosphorylation and acetylation in response to epidermal growth factor stimulation. Mol Cell 2000;5:905-15.

[51] Li J, Lin Q, Yoon HG, Huang ZQ, Strahl BD, Allis CD, et al. Involvement of histone methylation and phosphorylation in regulation of transcription by thyroid hormone receptor. Mol Cell Biol 2002;22: 5688-97.

[52] Crosio C, Heitz E, Allis CD, Borrelli E, Sassone-Corsi P. Chromatin remodeling and neuronal response: multiple signaling pathways induce specific histone $\mathrm{H} 3$ modifications and early gene expression in hippocampal neurons. J Cell Sci 2003;116:4905-14.

[53] Tsankova NM, Kumar A, Nestler EJ. Histone modifications at gene promoter regions in rat hippocampus after acute and chronic electroconvulsive seizures. J Neurosci 2004;24:5603-10.

[54] He H, Lehming N. Global effects of histone modifications. Brief Funct Genomic Proteomic 2003;2:234-43.

[55] Samaco RC, Nagarajan RP, Braunschweig D, LaSalle JM. Multiple pathways regulate $\mathrm{MeCP} 2$ expression in normal brain development and exhibit defects in autism-spectrum disorders. Hum Mol Genet 2004;13:629-39.

[56] Johnston MV, Mullaney B, Blue ME. Neurobiology of Rett syndrome. J Child Neurol 2003;18:688-92. 